\title{
Diferencias según género en la intención de emigración en médicos recién egresados
}

\author{
Gender differences in the intention of recent Peruvian medical graduates to emigrate
}

\author{
Reneé Pereyra-Elías ${ }^{1,2, a}$, Alessandra Cassana ${ }^{1,2, a}$, Edward Mezones-Holguín ${ }^{1,3, b}$, \\ Percy Mayta-Tristán ${ }^{1,3, c}$ \\ 'Escuela de Medicina, Universidad Peruana de Ciencias Aplicadas. Lima, Perú. \\ ${ }^{2}$ Sociedad Cientifica de Estudiantes de Medicina de la Universidad Peruana de Ciencias Aplicadas (SOCIEMUPC), Lima, Perú. \\ ${ }^{3}$ Instituto Nacional de Salud, Lima, Perú. \\ ${ }^{a}$ Estudiante de medicina, ${ }^{b}$ Médico epidemiólogo clínico, ${ }^{\mathrm{C}}$ Médico salubrista.
}

\begin{abstract}
Resumen
Introducción: La emigración médica en el Perú supone un importante problema en la búsqueda de la consolidación de un sistema de salud sostenible. Objetivos: Estimar la prevalencia de intención de emigración y sus factores asociados según género en médicos peruanos recién egresados. Diseño y lugar de estudio: Estudio analítico de corte transversal llevado a cabo en Lima, Perú, durante el año 2010. Participantes: Se incluyó a 289 médicos egresados de universidades de peruanas. Intervenciones: Se aplicó una encuesta anónima y autoadministrada que evaluaba la intención de emigración para laborar y sus factores asociados. Los datos fueron analizados con el paquete estadístico STATA 11.2; se utilizó un modelo lineal generalizado log-binomial como método de regresión de múltiples variables. Principales medidas de resultados: Intención de emigración autorreportada. Resultados: La prevalencia de intención de emigración fue de 42,1\%; esta fue mayor en el género masculino (50,0\% versus $36,4 \%$; $p<0,01)$. Un manejo intermedio/avanzado del idioma inglés (RP: 1,77; IC95\%: 1,04 a 2,98) y expectativas de ingreso económico mayor o igual a 3600 dólares americanos mensuales en los siguientes cinco años (RP: 1,55; IC95\%: 1,09 a 2,21) se encontraron asociados con la intención de emigrar en varones. En mujeres, estuvieron asociados el haber culminado la carrera sin retraso (RP: 1,66; IC95\%: 1,02 a 2,71) y proyectarse a ganar 3600 dólares o más (RP: 1,71; IC95\%: 1,14 a 2,57). Conclusiones: Existe una alta prevalencia de intención en emigración médica; el factor económico se asocia independientemente; los idiomas y la regularidad académica difieren según género.
\end{abstract}

Palabras clave: Migración internacional; salarios y beneficios; recursos humanos; médicos (Fuente: DeCS BIREME).

\begin{abstract}
Introduction: Medical emigration is a really important problem for Peru in order to consolidate a sustainable health system. Objectives: To determine the prevalence of emigration intention and associated factors according to gender in recently Peruvian graduated physicians. Design and setting: Cross-sectional, analytic study developed in Lima, Peru in 2010. Participants: Two hundred eightynine recently graduated physicians from Peruvian universities were included. Interventions: An anonymous and auto-administered questionnaire was handed to the study subjects to measure their intention to work abroad. Data was analyzed using the statistical package STATA 11.2, and a log-binomial generalized lineal model was used as regression model for multiple variables. Main outcome measures: Self-reported emigration intention. Results: Prevalence of intended emigration was $42.1 \%$, higher in males $(50.0 \%$ versus $36.4 \% ; p<0.01)$. For males, an intermediate/advanced level of English proficiency ( $\mathrm{PR}=1.77 ; 95 \% \mathrm{Cl}: 1.04-2.98$ ) and a monthly income expectation greater than or equal to 3600 U.S. dollars in five years ( $P R=1.55 ; 95 \% \mathrm{Cl}: 1.09-2.21)$ were associated with the intention of emigrating. In women there was association with fulfilling the career without delay (PR:1.66; 95\% Cl: 1.02-2.71) and expectation to win 3600 USD or more in five years (PR:1.71; 95\% Cl: 1.14-2.57). Conclusions: There was high prevalence of emigration intention in the studied population; the economic factor was associated independently. However, English proficiency and academic regularity differed by gender.
\end{abstract}

Keywords: International migration, wages and benefits, human resources, medical (Source: MeSH NLM).

An Fac med. 2013;74(3):211-6

\section{INTRODUCCIÓN}

La creciente tendencia de emigración de los profesionales de la salud desde los países en vías de desarrollo hacia países más desarrollados ha puesto de manifiesto la existencia de un fenómeno conocido como 'fuga de cerebros' (1-6). Norteamérica, Europa y Australia son los principales receptores, países en los cuales la cuarta parte de médicos que ejercen son extranjeros y, de estos, hasta $75 \%$ procede de naciones de bajos ingresos ${ }^{(2,7-9)}$.

Este hecho ha dado lugar a múltiples debates éticos y económicos respecto a la disminución de profesionales médicos que satisfagan las necesidades de atención integral en salud de la población del país de origen ${ }^{(10,11)}$. En ese sentido, la Organización Mundial de la
Salud (OMS) y otras entidades internacionales afirman que las demandas de recursos humanos de los países en vías de desarrollo se han incrementado debido a la necesidad de establecer sistemas de salud sostenibles para el perfil epidemiológico propio de cada nación, los cuales suponen alta morbilidad y mortalidad ${ }^{(9,10,12)}$. La polémica se agudiza, no solo por la alta proporción de médicos que dejan estos países, sino 
porque la mayoría de los emigrantes busca establecerse de modo definitivo en el país receptor, con el subsecuente retorno insuficiente para cubrir las necesidades de los países donde fueron formados ${ }^{(3,13-15)}$.

En dicho contexto, son varias las razones asociadas con la 'fuga de cerebros' reportadas en la literatura, destacando factores relacionados con una mejora en el ingreso económico ${ }^{(5,16-20)}$, oportunidades de desarrollo profesional y familiar, así como mejores condiciones laborales ${ }^{(1,5,21-24)}$.

Para el año 2005, el Perú ostentaba el 'factor de emigración' (número de médicos del país de origen ejerciendo en el país destino dividido entre el total de médicos ejerciendo en el país de origen) más alto de toda América del Sur y Central, tomando como países receptores a Estados Unidos, Canadá, Reino Unido y Australia ${ }^{(2)}$. En concordancia con estos resultados, el Ministerio de Salud de Perú (MINSA) reconoce que existe un número insuficiente de médicos para una atención sanitaria que permita la obtención de altos estándares de salud en el país, lo cual también se ve influenciado por el adolecer de falta de preparación de los recursos humanos en salud (RHUS) y la distribución centralista a nivel nacional de los mismos ${ }^{(22,25-27)}$. Se ha reconocido que Perú es uno de los países en crisis de RHUS, por lo que, mediante diversas estrategias, se ha intentado revertir esta situación, obteniéndose ciertos resultados positivos al reorganizarlos en el territorio nacional (incremento y redistribución); sin embargo, aún sigue encontrándose lejos de lo ideal ${ }^{(28)}$.

Uno de los aspectos que influencia en la decisión de emigrar es el género; se ha encontrado que para los varones priman los motivos económicos y de desarrollo profesional, mientras que para las mujeres la esfera familiar y el matrimonio constituyen el aspecto más importante ${ }^{(29,30)}$. En Perú no hemos encontrado estudios que evalúen diferencias según género en la intención de emigrar, y en general la inves- tigación sobre recursos humanos en salud es limitada, por lo cual ha sido propuesta como la primera prioridad de investigación en salud para el periodo 2009-14 ${ }^{(31,32)}$.

Por ello, el objetivo del estudio fue estimar la prevalencia de intención de emigración y sus factores asociados según género en médicos recién egresados de universidades peruanas en el 2010.

\section{MÉTODOS}

Se realizó un estudio de corte transversal en el mes de abril de 2010, en el que se incluyó a todos los médicos que asistieron al último día de la segunda elección regional de sedes para el Servicio Rural y Urbano Marginal de Salud (SERUMS), en Lima, previo consentimiento informado y autorización de los responsables del MINSA encargados de llevar dicho proceso. Se excluyó a los que no completaron la variable de interés y a los que habían estudiado la carrera fuera del Perú (figura 1).

Para la recolección de datos, se elaboró una cuestionario basado en un estudio previo desarrollado en Perú ${ }^{(1)}$, el cual exploraba aspectos generales como edad, sexo, estado civil, año de ingreso y egreso de la carrera, universidad de procedencia, presencia de familiares médicos, dominio del inglés, proyección económica mensual a cinco años, entre otros.

Los participantes fueron ubicados -por tres encuestadores previamente capacitados- en la sede donde realizaría la elección de vacantes del SERUMS, de los cinco turnos distribuidos en tres días; solo se obtuvo el permiso de las autoridades para encuestarlos en el último día. Previa explicación del objetivo del estudio, fueron invitados a participar a quienes aceptaron realizar la encuesta autoaplicada, previo consentimiento informado verbal. El tiempo que demoró su ejecución fue de 10 \pm 5 minutos.

Se definió como intención de migración a la respuesta 'Sî' ante la pregunta:
" ¿Usted piensa ejercer la carrera fuera del país?". A aquellos que manifestaron que sí, se les preguntó si retornarían al Perú a trabajar después de haber migrado.

Se preguntó además por el monto en soles (luego se convirtió a dólares, a tasa de cambio del día de la ejecución) que el médico aspiraba ganar mensualmente dentro de un periodo de cinco años, tomando en cuenta el tiempo de realización del SERUMS, la aplicación a la segunda especialización y el desarrollo de la misma (promedio de cinco años en total), con lo que se intentó tener una proyección del sueldo esperado; se consideró la especialidad como ya realizada.

Las encuestas fueron digitadas por los investigadores en una base de datos en Microsoft Excel $\AA^{\circledR}$ y fueron exportadas al paquete estadístico Stata v.11.2, en el cual se llevó a cabo el control de calidad.

Posteriormente, se realizó un análisis descriptivo usando frecuencias absolutas y relativas en el caso de las variables categóricas, y media y desviación estándar, en el caso de las variables numéricas. El análisis inferencial se desarrolló a nivel bivariado con la prueba chi cuadrado o prueba exacta de Fisher, según correspondía para evaluar la asociación entre la intención de emigrar con otras variables categóricas. Se usó la prueba de suma de rangos de Wilcoxon cuando se trató de variables numéricas (previa comprobación de su no normalidad con la prueba de Shapiro Wilk).

Se calculó las razones de prevalencia crudas y ajustadas con sus respectivos intervalos de confianza al 95\%, usando modelos lineales generalizados log-binomial, con el fin de evaluar la asociación entre los diferentes factores con la intención de emigración. Se evidenció que la variable género producía interacción con el manejo de idiomas; por lo que se optó por desarrollar un modelo para varones y otro para mujeres; en ambos casos se encontró convergencia adecuada. Se consideró un $\mathrm{p}<0,05$ como estadísticamente significativo. 


\section{RESULTADOS}

De un total de 361 médicos encuestados, se excluyó 40 por devolver las encuestas vacías, cinco por no responder la variable de interés y 27 por haber estudiado medicina en el extranjero, quedando 289 encuestas válidas para el análisis (figura 1).

La edad promedio fue $25,5 \pm 2,2$ años, $56,2 \%$ fueron mujeres, $93,4 \%$ estaban solteros y $6,9 \%$ tenían hijos. La mayoría $(73,0 \%)$ estudió en universidades privadas y solo $2,1 \%$ estudió fuera de Lima. Los médicos procedieron principalmente de la Universidad de San Martín de Porres (40,1\%), Universidad Nacional Mayor de San Marcos $(15,6 \%)$ y Universidad Ricardo Palma $(14,5 \%)$.

La mayoría $(63,6 \%)$ de los médicos terminó la carrera en el plazo esta- blecido, según la malla curricular de su universidad; $20,6 \%$ lo hizo con un año adicional, $8,0 \%$ dos años y hubo médicos que requirieron entre 3 a 12 años adicionales. Sobre el manejo del inglés, $4,5 \%$ declaró tener un conocimiento nulo, $19,7 \%$ básico, $46,4 \%$ intermedio y $29,4 \%$ avanzado. Más de la mitad $(54,0 \%)$ de los encuestados declaró tener familiares médicos; de ellos, $52,6 \%$ fueron familiares en primer grado o muy cercanos (padres, hermanos o abuelos).

Se observó diferencias estadísticamente significativas, según sexo, para intención de emigración, el manejo intermedio o avanzado del idioma inglés y la universidad de procedencia $(\mathrm{p}<0,05)$.

La prevalencia de intención de emigración para ejercer fuera del Perú fue 42,2\% (IC95\%: 36,5 a 47,9\%); sin em-

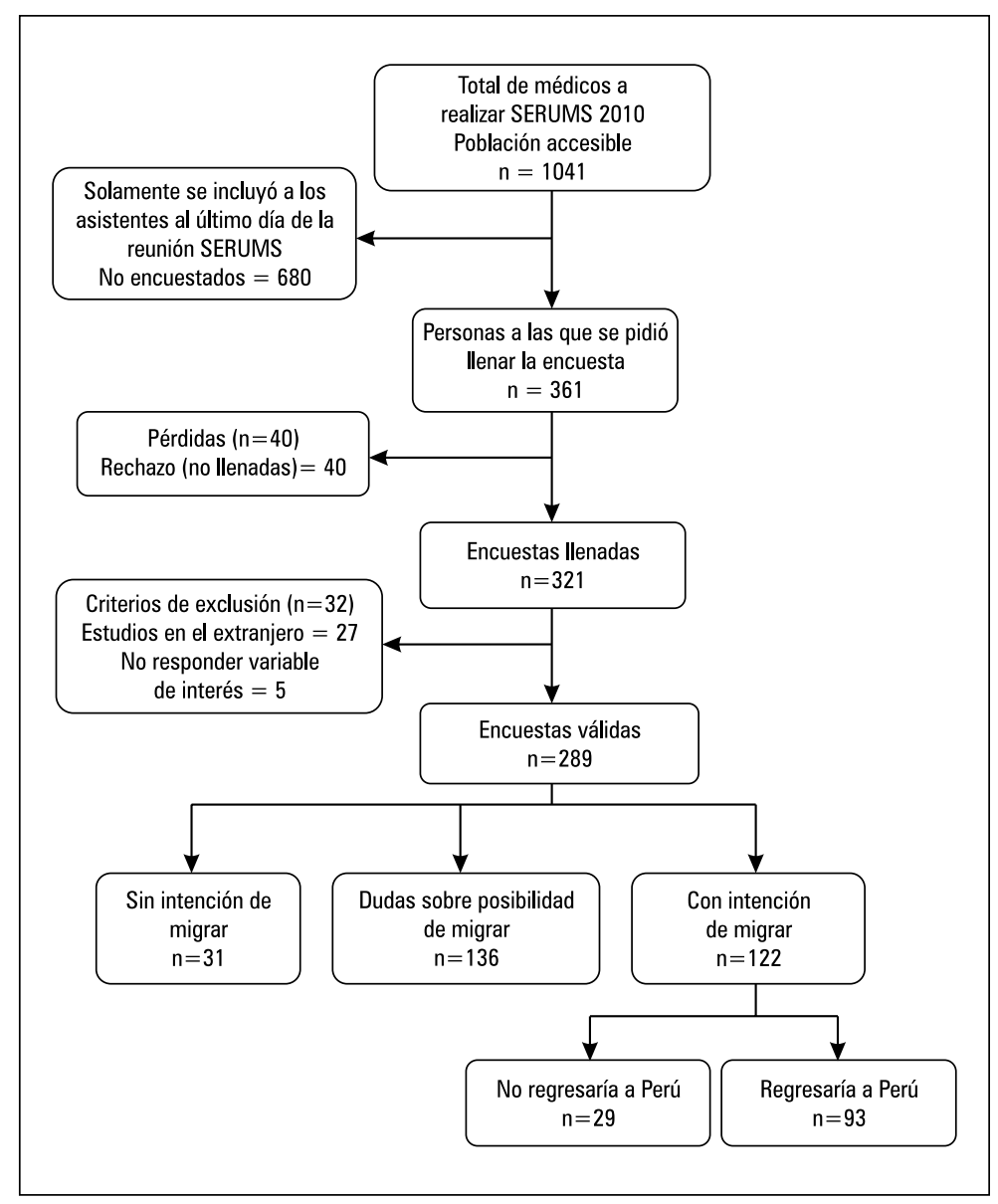

Figura 1. Flujograma de participación en el estudio.

bargo, $47,1 \%$ de los encuestados manifestó tener dudas sobre su posibilidad de migrar y solo $10,7 \%$ afirmó que no emigraría para ejercer la carrera. De aquellos que manifestaron su intención de emigrar, 23,6\% (29/122) refirió que no regresaría al Perú (figura 1).

En el análisis bivariado, no se encontró asociación con la edad, tener hijos, ser soltero, tener familiares médicos y haber estudiado en una universidad pública o privada. Proyectarse a tener un ingreso económico alto se encontró como un factor asociado para ambos sexos. Tener un manejo intermedio o avanzado del idioma inglés se asoció a la intención de emigración en los varones mas no en las mujeres y concluir la carrera en el tiempo establecido se encontró asociado en las mujeres mas no en los varones; esto, por una interacción producida por el género, la cual fue confirmada en el análisis de regresión de múltiples variables (tabla I).

Al respecto, inicialmente, se llevó a cabo un modelo único donde se tomó al género como una variable de control más; posteriormente, se generó variables de interacción entre el género con el dominio del inglés, así como, entre el género con el haber concluido la carrera sin retraso, observándose que eran significativas. En base a ello, se generó un modelo individual para cada género.

En el modelo desarrollado para los varones, las variables que se asociaron a la intención de emigrar para ejercer la carrera fueron el tener un manejo intermedio o avanzado del idioma inglés $(\mathrm{RP}=1,77$; IC95\%: 1,04 a 2,98$)$ $y$ el tener expectativas de un ingreso económico mayor o igual a 3600 dólares americanos mensuales en los próximos cinco años (RP: 1,55; IC95\%: 1,09 a 2,21). En el caso de las médicos mujeres, fueron el haber culminado la carrera en el tiempo estipulado por su universidad (RP: 1,66; IC95\%: 1,02 a 2,71) y el proyectarse a ganar 3600 dólares americanos o más en los próximos cinco años (RP: 1,71; IC95\%: 1,14 a 2,57). Ambos modelos convergieron adecuadamente. 
Tabla 1. Factores asociados, según sexo, con la intención de emigración en médicos peruanos recién egresados, Lima 2010. Análisis bivariado.

\begin{tabular}{|c|c|c|c|c|c|c|c|c|c|c|c|c|}
\hline \multirow{3}{*}{ Variable } & \multicolumn{6}{|c|}{ Varón } & \multicolumn{6}{|c|}{ Mujer } \\
\hline & \multirow[t]{2}{*}{ Total } & \multicolumn{2}{|c|}{$\begin{array}{l}\text { Intención de } \\
\text { emigración }\end{array}$} & \multirow[t]{2}{*}{$\mathrm{RP}$} & \multirow[t]{2}{*}{ IC95 } & \multirow[t]{2}{*}{$p$} & \multirow[t]{2}{*}{ Total } & \multicolumn{2}{|c|}{$\begin{array}{l}\text { Intención de } \\
\text { emigración }\end{array}$} & \multirow[t]{2}{*}{$\mathrm{RP}$} & \multirow[t]{2}{*}{ IC95\% } & \multirow[t]{2}{*}{$\mathrm{p}$} \\
\hline & & $\mathrm{n}$ & $(\%)$ & & & & & $\mathrm{n}$ & $(\%)$ & & & \\
\hline \multicolumn{13}{|l|}{ Edad } \\
\hline$>25$ & 50 & 21 & $(42,0)$ & \multirow[t]{2}{*}{0,76} & \multirow[t]{2}{*}{$0,52-1,11$} & \multirow[t]{2}{*}{$0,143^{\mathrm{a}}$} & 55 & 15 & $(22,3)$ & \multirow[t]{2}{*}{0,66} & \multirow[t]{2}{*}{$0,41-1,08$} & \multirow[t]{2}{*}{$0,083^{\mathrm{a}}$} \\
\hline$\leq 25$ & 74 & 41 & $(55,4)$ & & & & 107 & 44 & $(41,2)$ & & & \\
\hline \multicolumn{13}{|l|}{ Tiene hijos } \\
\hline Sí & 8 & 3 & $(37,5)$ & \multirow[t]{2}{*}{0,74} & \multirow[t]{2}{*}{$0,30-1,84$} & \multirow[t]{2}{*}{$0,717^{b}$} & 12 & 1 & $(8,30)$ & \multirow[t]{2}{*}{0,22} & \multirow[t]{2}{*}{$0,03-1,42$} & \multirow[t]{2}{*}{$0,057^{b}$} \\
\hline No & 118 & 60 & $(50,9)$ & & & & 150 & 58 & $(38,7)$ & & & \\
\hline \multicolumn{13}{|l|}{ Estado civil } \\
\hline Casado/conviviente & 6 & 2 & $(33,3)$ & 0,66 & $0,21-2,06$ & $0,680^{b}$ & 13 & 2 & $(15,4)$ & 0,40 & $0,11-1,46$ & $0,136^{b}$ \\
\hline Soltero & 120 & 61 & $(50,8)$ & & & & 149 & 57 & $(38,3)$ & & & \\
\hline Familiares médicos & & & & & & & & & & & & \\
\hline Primer grado & 35 & 18 & $(51,4)$ & 1,12 & $0,74-1,70$ & $0,604^{c}$ & 46 & 16 & $(34,8)$ & 1,01 & $0,61-1,69$ & $0,956^{b}$ \\
\hline Segundo grado & 28 & 16 & $(57,1)$ & 1,24 & $0,82-1,88$ & 0,310 & 46 & 19 & $(41,3)$ & 1,20 & $0,75-1,93$ & 0,440 \\
\hline No & 63 & 29 & $(46,0)$ & & & & 70 & 24 & $(34,3)$ & & & \\
\hline Requirió más tiempo para acabar I & & & & & & & & & & & & \\
\hline Sí & 43 & 19 & $(44,2)$ & 0,83 & $0,56-1,23$ & $0,347^{\mathrm{a}}$ & 61 & 16 & $(26,2)$ & 0,62 & $0,39-1,00$ & $0,046^{\mathrm{a}}$ \\
\hline No & 83 & 44 & $(53,0)$ & & & & 98 & 41 & $(41,8)$ & & & \\
\hline Inglés & & & & & & & & & & & & \\
\hline Intermedio o Avanzado & 88 & 51 & $(58,0)$ & 1,84 & $1,11-3,03$ & $0,011^{\mathrm{a}}$ & 130 & 49 & $(37,8)$ & 1,21 & $0,69-2,11$ & $0,545^{\mathrm{a}}$ \\
\hline No -básico & 38 & 12 & $(31,6)$ & & & & 32 & 10 & $(31,3)$ & & & \\
\hline Universidad de procedenci & & & & & & & & & & & & \\
\hline Privada & 84 & 47 & $(56,0)$ & 1,47 & $0,96-2,26$ & $0,059^{\mathrm{a}}$ & 127 & 45 & $(35,4)$ & 0,89 & $0,55-1,42$ & $0,612^{\mathrm{a}}$ \\
\hline Pública & 42 & 16 & $(38,1)$ & & & & 35 & 14 & $(40,0)$ & & & \\
\hline Ingreso económico proyecta & & & & & & & & & & & & \\
\hline 10000 o más soles & 51 & 33 & $(64,7)$ & 1,77 & $1,13-2,78$ & $0,013^{c}$ & 47 & 23 & $(48,9)$ & 1,64 & $1,03-2,62$ & $0,039^{\circ}$ \\
\hline 5 000-9 999 soles & 30 & 12 & $(40,0)$ & 1,09 & $0,60-1,98$ & 0,769 & 38 & 12 & $(31,6)$ & 1,06 & $0,58-1,92$ & 0,853 \\
\hline$<5000$ soles & 41 & 15 & $(36,6)$ & & & & 67 & 20 & $(29,9)$ & & & \\
\hline
\end{tabular}

a: $\mathrm{Chi}^{2}$, b: Prueba exacta de Fisher; c: Modelo lineal generalizado log-binomial.

\section{DISCUSIÓN}

La intención de emigración en nuestro estudio fue $42,2 \%$, similar a lo encontrado por Mayta-Tristán y col. en el 2008 y 2010, donde se trabajó con una población de internos de una universidad pública peruana y estudiantes de medicina de 19 universidades latinoamericanas, respectivamente ${ }^{(1,14)}$.

Por otra parte, se halló que uno de cada cuatro médicos no regresaría a Perú. Este hallazgo es similar a lo observado en estudios realizados en médicos libaneses y por la experiencia previa en América Latina ${ }^{(12,14)}$. El considerable porcentaje de personas que no regresaría deriva de las razones por las cuales se migró en primera instancia, que son halladas en el país de destino y no en el de origen: motivos económicos, sociales y de desarrollo profesional ${ }^{(1,5,9-11,16,17)}$. Por otro lado, los médicos que migrarían y posteriormente retornarían podrían estar motivados por limitaciones percibidas para el desarrollo de una especialización en Perú, situación que sería importante de evaluar en estudios futuros.

Al comparar la intención de ambos sexos, se evidencia que la de los varones es mayor. Estudios previos en Libia encuentran la misma tendencia, la cual se relaciona con la mayor independencia que supone el género masculino ${ }^{(9,13)}$. Sin embargo, estos resultados contrastan con la experiencia en mujeres médicas de México, en la que se en- contró que son más propensas a migrar a lugares alejados en el interior del país para ejercer ${ }^{(30)}$. Del mismo modo, un estudio en médicos peruanos que evaluó su intención de migración interna para trabajar no encontró diferencias según género ${ }^{(27)}$. Nuestro estudio demuestra que esta tendencia cambia en términos de emigración para los médicos peruanos.

Los factores asociados con la intención de migración incluyen características académicas y económicas. En el análisis bivariado, se encontró tendencia a la asociación con ser joven (menos de 25 años) y no tener hijos. Esta relación, que fue encontrada también en investigación previa ${ }^{(3,9,12,23)}$, puede ser explicada por las obligaciones o respon- 
sabilidades familiares que supone presentar estas variables. Para las mujeres, se halló asociación entre la variable de respuesta y el haber terminado la carrera en los años estipulados por la malla curricular. Es probable que esto se deba a buen desempeño académico que las condujo a culminar la carrera a tiempo, lo cual ha sido descrito en investigación previa ${ }^{(33)}$.

Por otro lado, la asociación encontrada con el manejo intermedio y avanzado del idioma inglés y la intención de migración para el sexo masculino podría deberse a que el grueso de países receptores de profesionales de la salud migrantes tiene al inglés como idioma principal, tal es el caso de Estados Unidos, Reino Unido, Australia y Canadá ${ }^{(2,3,8,16)}$. Sin embargo, el país probable de destino no fue evaluado en este estudio. Por otro lado, una investigación previa muestra que España es el principal destino para la población médica peruana, país que además tiene a Perú como principal origen de importación de médicos ${ }^{(1,34)}$, por lo que aparentemente el manejo del inglés no sería tan determinante para una posible emigración.

A diferencia del estudio realizado solo con internos de una universidad pública peruana ${ }^{(1)}$, nosotros incluimos médicos ya colegiados, provenientes tanto de universidades públicas como privadas. Sin embargo, no se encontró diferencias entre un grupo y otro respecto de la intención de migración. Los estudiantes de universidades privadas, en su mayoría, eran de un estrato socioeconómico medio-alto, lo cual podría suponer una mayor expectativa a emigrar, sea por sus características intrínsecas o por la accesibilidad o facilidad que tienen para hacerlo y continuar su desarrollo profesional en el extranjero. Sin embargo, esta asociación no fue observada en la presente investigación.

Es importante remarcar que existió diferencia entre el tener intención de migrar y el poder concretar la misma ${ }^{(11)}$. El hecho de que no existan diferencias entre los estudiantes de universidades públicas y privadas podría explicarse sobre la base de haber medido solamente la mera intención de migrar, mas no el hecho consumado de hacerlo. Es probable que si se evalúa la migración como cuestión fáctica, se encuentre diferencias entre estos dos grupos, por las facilidades de acceso antes mencionadas.

La expectativa de un sueldo alto fue un hallazgo independiente del sexo. Su importancia, en base a la comparación con el antecedente peruano de intención de migración ${ }^{(1)}$, radica en el incremento considerable del punto de corte de asociación entre la intención de migración y el monto que se aspira a ganar mensualmente. Mayta-Tristán y col. encontraron que aspirar a ganar 2150 dólares americanos o más estaba asociado a la intención de migración ${ }^{(1)}$; mientras que en el presente estudio se encontró asociación con aspirar a ganar un monto mayor a 3600 dólares americanos. Es poco probable que en menos de tres años (tiempo transcurrido entre un estudio y otro) la situación económica y laboral del país haya sufrido cambios que expliquen esta variación. Sin embargo, esto podría tener una explicación basada en los sueldos que perciben los internos en Perú, que fluctúan entre 150 y 300 dólares americanos, a diferencia de los 1100 a 1800 dólares de un médico recién graduado que realiza el SERUMS. El incremento de las expectativas salariales de los sujetos de estudio puede deberse a la diferencia en los montos percibidos: mientras mayor sea este, mayor será la expectativa de ingreso monetario. Es importante mencionar también que, si bien existió una mejora económica en el país - cambio del producto bruto interno per cápita de 4400 a 5280 dólares en ese periodo - esto no se reflejó en mejoras salariales en el sector salud ${ }^{(35)}$.

Si bien hay autores que apoyan el derecho de libre migración definitiva de médicos a países desarrollados para el cumplimiento de sus metas personales ${ }^{(5,36)}$, el beneficio individual debe ser sopesado con las consecuencias que involucra esta decisión en cuanto al bien y prosperidad de una sociedad $(15,37)$. De hecho, en el año 2005, se aprobó la emigración de profesionales de la salud como uno de los desafíos en Recursos Humanos ${ }^{(38)}$, donde se planteaba, entre otras medidas, regular dichos desplazamientos para garantizar una atención equitativa en la población; las mismas que fueron aprobadas como metas de los Ministerios de Salud de las Américas en la 27ํㅡㄹ Conferencia Panamericana de la Salud ${ }^{(39)}$.

En Perú, existe una marcada inequidad en la distribución de profesionales de la salud ${ }^{(40)}$ que se acentúa por el proceso de migración a otros países, por lo que se ha tomado ciertas medidas amortiguadoras, como la beca de repatriación implementada por la Universidad Peruana Cayetano Heredia, mediante la cual se favorece el retorno a Perú de científicos destacados ${ }^{(41)}$. Estrategias que dibujen el mismo objetivo deben ser desarrolladas para los médicos a nivel de Estado, con el fin de lograr su estadía o retorno y retención en el país.

Este estudio presenta ciertas limitaciones. El haber utilizado una muestra no probabilística no permite extrapolar los resultados a la población total de médicos recién colegiados de Perú. Por otro lado, evaluar los principales motivos y destinos de migración hubiese sido de gran importancia, además de conocer la intensidad de la intención de migración (evaluando los conocimientos que tienen acerca de cómo migrar) de los mismos o las facilidades que tenían para cumplir con este propósito. En última instancia, el hecho de que el estudio sea de corte transversal no permite establecer relaciones causales, sino solamente asociaciones.

En conclusión, los médicos peruanos recién colegiados presentan una prevalencia considerable de intención de emigración. Los factores asociados son declarar tener un manejo del inglés a nivel intermedio o avanzado en los varones y haber terminado la carrera en los años estipulados por la currícula de la universidad en las mujeres. Proyec- 
tarse a tener un ingreso económico mayor o igual a 3600 dólares americanos mensuales en los próximos cinco años se muestra como un factor asociado independiente del género.

Los profesionales de la salud, en especial los médicos, son recursos humanos esenciales en un país en vías de desarrollo como el Perú. Para evitar esta problemática, es preciso instituir políticas de trabajo que impliquen mejoras salariales, mejoras en las condiciones de trabajo y en las condiciones de vida en general. Conocer mejor la prevalencia de intención de migración en la población total de médicos de Perú es importante para tener una mejor visión del panorama y una perspectiva más clara de las medidas a aplicar para revertir esta situación.

\section{AGRADECIMIENTOS}

Agradecemos a Anny Huillca Briceño y a Melissa Arce Oyola por su gentil colaboración en la recolección de datos.

\section{REFERENCIAS BIBLIOGRÁFICAS}

1. Mayta-Tristán P, Dulanto-Pizzorni A. Prevalencia y factores asociados con la intención de emigración en internos de medicina de una universidad pública, Lima 2007. Rev Peru Med Exp Salud Publica. 2008;25(3):274-8.

2. Mullan $F$. The metrics of the physician brain drain N Engl J Med. 2005;353(17):1810-18.

3. Akl E, Maroun N, Major S, Afif C, Abdo A, Choucair $\mathrm{J}$, et al. Post-graduation migration intentions of students of Lebanese medical schools: a survey study. BMC Public Health. 2008;8:191

4. Zweig D, Siu C, Han D. Redefining the brain drain: China's 'diaspora option'. Sci Technol So. 2008;13(1):1-33

5. Aly Z, Taj F. Why Pakistani medical graduates must remain free to emigrate. PloS Med. 2008;5(1):e2.

6. Forstenlechner I. Brain drain in developed countries: can governments do anything to bring expatriates back? Public Policy Adm. 2010;25(2):156-74

7. Talati J, Pappas G. Migration, medical education, and health care: a view from Pakistan. Acad Med 2006;81(12):S55-S62

8. Arah OA, Ogbu UC, Okeke CE. To poor to live, too rich to stay: developmental and global health correlates of physician migration to the United States, Canada, Australia, and the United Kingdom. Am J Public Health. 2008;98(1):148-54.

9. AkI E, Maroun N, Major S, Chahoud B, Schunemann HJ. Graduates of Lebanese medical schools in the United States: an observational study of international migration of physicians. BMC Health Serv Res. 2007;7:49.
10. Dwyer J. What's wrong with the global migration of health care professionals? Individual rights and international justice. Hastings Cent Rep. 2007;37(5):36-43.

11. Chen LC, Boufford JI. Fatal flows--doctors on the move. N Engl J Med. 2005;353(17):1850-2.

12. Organización Mundial de la Salud. Perfil mundial de los trabajadores sanitarios. Informe mundial sobre la salud. Ginebra: OMS; 2006.

13. Benamer HT, Bredan A, Bakoush O. The Libyan doctors' brain drain: an exploratory study. BMC Res Notes. 2009;2:242.

14. Mayta-Tristán P, Carbajal-Gonzalez D, MezonesHolguín E, Mejia CR, Pereyra-Elias R, VillafuerteGálvez J, et al Perspectivas profesionales e intención de emigración de los estudiantes de Medicina de nueve países de Latinoamérica, 2008: Estudio preliminar. CIMEL. 2010;15(1):3-8.

15. Mayta-Tristán $P$, Dulanto-Pizzornni A. Emigración médica: lo público frente a lo individual. Rev Peru Med Exp Salud Publica. 2008;25(4):447-8.

16. Vujicic M, Zurn P, Diallo K, Adams O, Dal Poz M. The role of wages in the migration of health care professionals from developing countries. Hum Resour Health. 2004;2(1):3.

17. Syed NA, Khimani F, Andrades M, Ali SK, Paul R. Reason for migration among medical students from Karachi. Med Educ. 2008;42(1):61-8.

18. Akl E, Maroun N, Major S, et al. Why are you draining your brain? Factors underlying decisions of graduating Lebanese medical students to migrate. Soc Sci Med. 2007;64(6):1278-84.

19. Bernardini-Zambrini D, Barengo N, Bardach A, Hanna M, Núñez JM. ¿Migrar o no migrar? ¿Qué pasará con nuestra próxima generación de médicos? Estudio sobre causas y motivos en estudiantes avanzados de medicina en 11 Universidades de España. Aten Primaria. 2011;43(5):222-6.

20. Pardo K, Andia M, Rodriguez A, Perez W, Moscoso B. Remuneraciones, beneficios e incentivos laborales percibidos por trabajadores del sector salud: análisis comparativo entre el Ministerio de Salud y la Seguridad Social, 2009. Rev Peru Med Exp Salud Publica. 2011;28(2):342-51.

21. Mullan F. Doctors for the world: Indian physician emigration. Health Affairs. 2006;25(2):380-93

22. Perú, Ministerio de Salud. Profesionales de la salud del sector salud Perú: 1980 - 2002. Lima: MINSA; 2008

23. Rosales-Martínez Y, Nigenda G, Galárraga O, RuizLarios JA. Expectativas de migración internacional en estudiantes de enfermeria de México, Distrito Federal. Salud Publica Mex. 2010;52(3):244-53.

24. Hernández T, Ortiz Gómez Y. La migración de médicos en Venezuela. Rev Panam Salud Publica. 2011;30(2):177-81.

25. Carrasco V, Lozano E, Velásquez E. Análisis actual y prospectivo de la oferta y demanda de médicos en el Perú 2005-2011. Acta Med Peru. 2008;25(1):22-29.

26. Zevallos L, Pastor R, Moscoso B. Oferta y demanda de médicos especialistas en los establecimientos de salud del Ministerio de Salud: brechas a nivel nacional, por regiones y tipo de especialidad. Rev Peru Med Exp Salud Publica. 2011;28(2):177-85.

27. Mayta-Tristán P, Mejia CR, Riega-López P, Rojas-Mezarina L, Posso M, Mezones-Holguín E. Proyección de trabajo en el interior del pais y factores asociados en médicos recién colegiados de Lima, Perú 2010. Rev Peru Med Exp Salud Publica. 2011;28(2):186-93

28. Dayrit MM, Dolea C, Dreesch N. Addressing the Human Resources for Health crisis in countries:
How far have we gone? What can we expect to achieve by 2015 ? Rev Peru Med Exp Salud Publica. 2011;28(2):327-36

29. Duverne A, Carnet D, d'Athis P, Quantin C. French doctors working in Great Britain: a study of their characteristics and motivations for migration. Rev Epidemiol Sante Publique. 2008;56(5):360-73.

30. Harrison ME. Female physicians in Mexico: migration and mobility in the lifecourse. Soc Sci Med. 1998;47(4):455-68.

31. Yagui M, Vargas J. La agenda nacional de investigación en recursos humanos en salud: generando evidencias para mejorar las competencias en el sector salud. Rev Peru Med Exp Salud Publica. 2011;28(2):175-6.

32. Curisinche M, Yagui M, Castilla T, Cabezas C, Escalante G, Casas M, Lucero J. Proceso de construcción de la agenda nacional de investigación sobre recursos humanos en salud (RHUS) en el Perú, 2011 - 2014. Rev Peru Med Exp Salud Publica. 2011;28(2):372-81.

33. Kaushik M, Roy A, Bang AA, Mahal A. Quality of medical training and emigration of physicians from India. BMC Health Serv Res. 2008;8:279.

34. Bernardini-Zambrini DA, Garcia-Gutiérrez JF, Mayta-Tristán P. Migración de médicos peruanos a España, 2005-2009. Rev Peru Med Exp Salud Publica. 2011;28(4):688-99.

35. Estadisticas [Internet]. Lima: Ministerio de Economia y Finanzas; 2013. Política económica y social: principales indicadores macroeconómicos; 2010 [citado 18 Abril 2013]. Disponible en: http://www. mef.gob.pe/index.php?option=com_content\&view $=$ article\&id=266\&ltemid=100606.

36. Khalid U, Saleem T. Medical graduates and migration--a perspective on the egression of human capital from Pakistan. J Pak Med Assoc. 2011;61(4):408-9

37. Ahmad OB. Managing medical migration from poor countries. BMJ. 2005;331(7507):43-5.

38. Novick M. Desafíos de la Gestión de los Recursos Humanos en Salud: 2000-2015. Washington DC: Organización Panamericana de la Salud; 2006.

39. Organización Panamericana de la Salud. 27.a Conferencia Sanitaria Panamericana 59.a Sesión del Comité Regional: Resolución CSP27.R10 - Política y estrategia regionales para la garantía de la calidad de la atención sanitaria, incluyendo la seguridad del paciente. Washington DC: Organización Panamericana de la Salud; 2007.

40. Organización Panamericana de la Salud. Migración de recursos humanos en salud. Estudio Subregión Andina / Organización Panamericana de la Salud, Red Andina de Asociaciones de Facultades y Escuelas de Medicina. Lima: Organización Panamericana de la Salud; 2006.

41. Guerra H. La beca de retorno de la Universidad Peruana Cayetano Heredia. Rev Peru Med Exp Salud Publica. 2010;27(3):428-31.

Artículo recibido el 14 de febrero de 2013 y aceptado para publicación el 5 de mayo de 2013.

Financiamiento: Autofinanciado.

Conflictos de interés: Ninguno.

Correspondencia:

Dr. Percy Mayta-Tristán

Dirección: Av. Brasil \#2169, Dpto 802, Lima 11, Perú.

Teléfono: (511) 987-532-133

Correo electrónico: percy.mayta@upc.edu.pe 